\title{
Defect depth determination in a CFRP structure using TSR technique
}

\author{
by B. Oswald-Tranta*, A.Maier**, R. Schledjewski** \\ ${ }^{*}$ Chair of Automation, University of Leoben, Austria, beate.oswald@unileoben.ac.at \\ ${ }^{*}$ Chair of Processing of Composites, University of Leoben, alexander.maier@unileoben.ac.at \\ ralf.schledjewski@unileoben.ac.at
}

\begin{abstract}
TSR technique can be well used to investigate the response to a short heating pulse in the time domain. As the time, when the heat starts to accumulate above a defect, is characteristic for its depth, this technique is appropriate for creating a 3D map of the sample. A CFRP structure with different number of layers and with Teflon inserts was used to demonstrate this technique. In order to calculate the depth of the artificial delaminations and the thickness of the layers, the starting time of the accumulation is determined for each pixel separately, based on the maximum of the 2nd derivative of the fitted temperature-time function and a 3D map of the structure generated.
\end{abstract}

Keywords: thermography, flash heating, defect detection, defect depth, CFRP, TSR

\section{Introduction}

In the last years active thermography inspection has been well established as a non-destructive testing technique for detecting internal defects in materials. Usually a pulse or a sinusoidal heating is applied to the surface and the response that means the temperature change in time is recorded and analysed. The specimen's answer to a sinusoidal heating is evaluated by the lock-in technique [1]. The answer to a pulse heating can be either analysed in the frequency domain using the PPT method [2] or in the time-domain by the Thermographic Signal Reconstruction (TSR) [3]. These methods can be not only used to detect subsurface defects, but also, with some restriction, to determine their depth. In the case of lock-in and PPT methods, the so-called blind frequency is searched, at which the phase contrast between the sound part and the defect is diminishing $[4,5,6]$. In the case of the TSR technique, after a preliminary step of a polynomial fitting, the derivatives of the temporal temperature change are used. As it has been also shown [7], in this way an early detection of the defects can be achieved.

In this paper the TSR technique is used to create a 3D map of a CFRP sample. The specimen contains artificial delaminations due to Teflon inserts. Additionally, it has been manufactured in a way that layers overlap each other only partially, creating areas with different thicknesses.

\section{Creation of the CFRP sample}

A CFRP structure with artificial defects has been created using the Vacuum Infusion Molding Process. Basic vacuum infusion is an easy method for manufacturing endless fiber reinforced composite parts. Therefore, only a simple, geometry-defining mold, vacuum bag, vacuum pump, resin and reinforcements are needed. In this work, for test specimen production, a slightly modified vacuum infusion was used. The slightly modified concept of vacuum infusion is to spread the resin in large areas by the help of flowing agents and to impregnate the reinforcements through the thickness. The benefit of this mechanism is that the resin has only a short infusion path through the thickness of the manufactured parts, rather than a long way through the length of the parts. The maximum possible diameters (length, width and height) are dependent on the flow-resistance and the type of resin, as well as the reinforcement structures, the component thickness and the effectiveness of the vacuum $[8,9,10]$. Typically, with vacuum infusion, composite parts with a fiber percentage up to $60 \%$ are possible [11]. [12]In Fig. 1 a typical vacuum infusion set-up is shown [12]. 


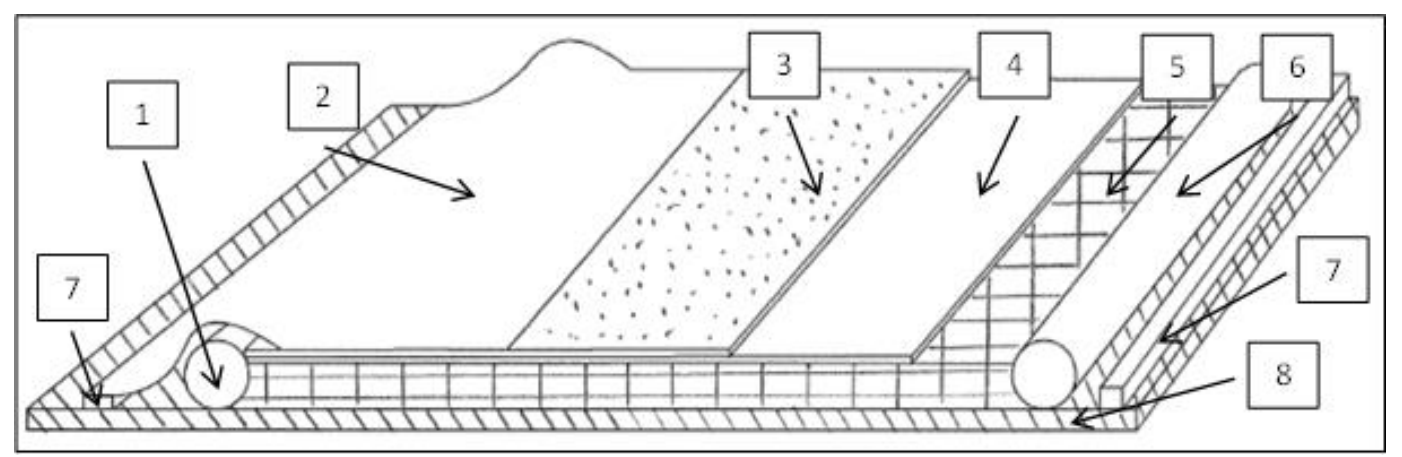

Figure 1: Schematic drawing of a typical vacuum infusion construction; mold assembly: 1, inlet; 2, vacuum bag; 3, distribution medium; 4, peel ply; 5, reinforcement; 6, outlet; 7, vacuum seal; 8, mold die; Maier A., et al.[12] (2014)

The test sample structure consists of 5 carbon fibre layers, which are rotated to each other and in this way there are regions, where only 1,2, 3, 4 and then 5 layers lie on each other, representing different thick samples in the corners (Fig.2). Additionally, couple of Teflon inserts have been placed between the layers, representing artificial delaminations in different depths.
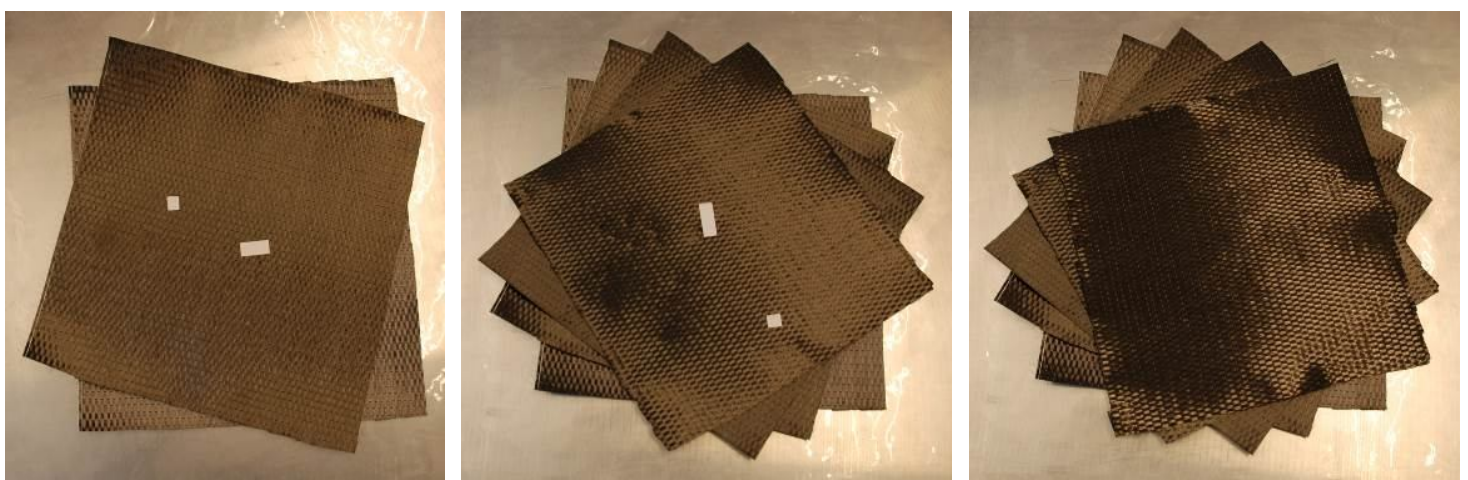

Figure 2: Five carbon fibre layers before applying vacuum infusion to create the CFRP sample. Additionally, as artificial defects, Teflon inserts have been placed between the layers.

The composite plates were manufactured from unidirectional (UD) carbon fabrics (style $4423 \mathrm{~K}$ Aero) and epoxy resin by the SCRIMP method. The epoxy resin used was EPIKOTETM Resin MGS® RIMR 135, and the hardener was EPIKOTETM Curing Agent MGS® RIMH 135, before the manufacturing process, stored at room temperature. The mixing ratio for resin-to-hardener in weight was 10:3. The composite plates were cured for $24 \mathrm{~h}$ at room temperature $\left(23^{\circ} \mathrm{C}\right)$ and under vacuum at a constant -900 mbar pressure.

The testing sample consists of 5 layers, each layer had dimensions of $400 \mathrm{~mm} \times 400 \mathrm{~mm}$ and a thickness of about $0.5 \mathrm{~mm}$ and between each layer small Teflon pieces with various dimensions were positioned to simulate delamination. Due to this stacking, a thickness of the testing specimen of $2.5 \mathrm{~mm}$ was reached.

\section{Inspection in transmission mode}

In the case of investigation in transmission mode, the temperature increase of a sample after an instantaneous heating by adiabatic conditions using a one-dimensional model can be written as $[13,14]$

$$
T_{\text {transm }}(z=d, t)=\frac{Q \kappa}{\lambda d}\left[1+2 \sum_{n=1}^{\infty}(-1)^{n} \exp \left(\frac{-n^{2} \pi^{2}}{d^{2}} \kappa t\right)\right]
$$

where $d$ is the thickness, $\kappa$ is the thermal diffusivity, $\lambda$ thermal conductivity of the material and $Q$ is the applied heat amount. After normalising the temperature increase with

$$
T_{\text {end }}=\frac{Q \kappa}{\lambda d}
$$

one obtains 


$$
V_{\text {transm }}(z=d, t)=\frac{T_{\text {transm }}(z=d, t)}{T_{\text {end }}}=1+2 \sum_{n=1}^{\infty}(-1)^{n} \exp \left(\frac{-n^{2} \pi^{2}}{d^{2}} \kappa t\right)
$$

When the temperature reaches the half of its maximum value, that means $V_{\text {transm }}=0.5$, then a time $t_{1 / 2}$ can be specified. Based on this half time $t_{1 / 2}$, the thickness can be calculated [14] by

$$
d=\pi \sqrt{\frac{\kappa t_{1 / 2}}{1.38}}
$$

For the transmission measurement of the CFRP structure a flash heating pulse with $1 \mathrm{~ms}$ (full width half maximum) and with energy of $6 \mathrm{~kJ}$ from one xenon flash lamp is applied to one side of the sample. The temperature increase on the other side of the sample is recorded by a commercially available cryogenically cooled $320 \times 256$ pixel InSb camera [15], operating at $385 \mathrm{~Hz}$ in the $1.5-5 \mu \mathrm{m}$ spectral range. Fig. 3 shows measurement results, the normalised temperature increase after the flash heating is depicted for five points lying in regions with different number of carbon fiber layers.

Fig.4 shows a 3D depth map of the sample calculated from the transmission measurement. The Teflon inserts represent a barrier to the heat flow, therefore in the 3D map they occur as they would be much thicker than the sample itself. With the transmission measurement the whole thermal thickness of the sample, that means the sum of the layers and inserts can be determined. The defects, independently on their depth, can be made excellently visible, but there is no possibility to determine their depths.

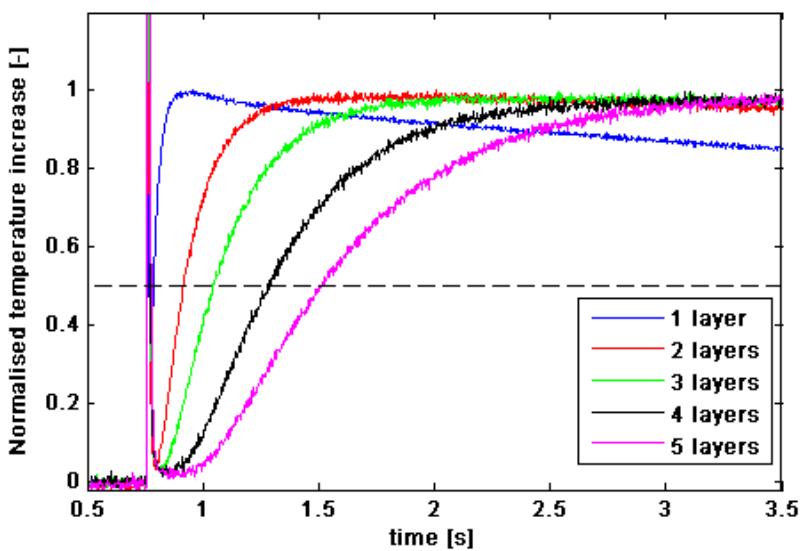

Figure 3: Normalised temperature increase in transmission measurement for 5 different points of the CFRP sample.

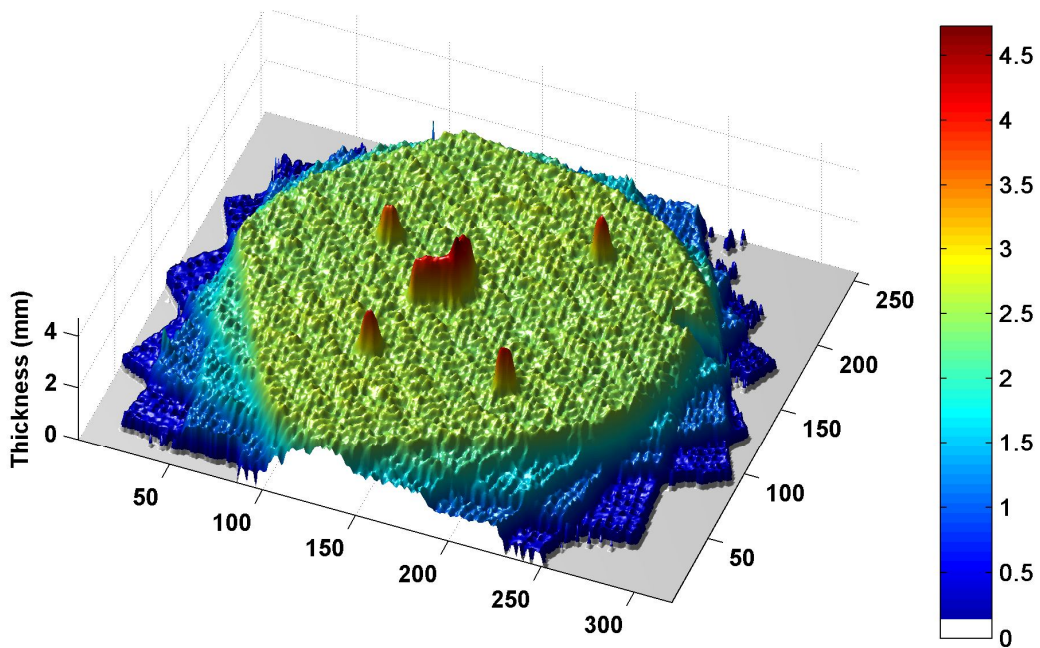

Figure 4: 3D map of the sample calculated from transmission measurement; the Teflon inserts, as thermal barriers to the heat flow, become visible due to the seemingly high thickness values 


\section{Inspection in reflection mode}

In reflection mode the heating pulse is applied on the same side of the sample where also the temperature increase is recorded. In this way the heat flow, reflected from the back side of sample and from the defects is investigated. The temperature increase after an instantaneous heating by adiabatic conditions is given $[13,14]$ by

$$
T_{\text {refl }}(z=0, t)=T_{\text {end }}\left[1+2 \sum_{n=1}^{\infty} \exp \left(\frac{-n^{2} \pi^{2}}{d^{2}} \kappa t\right)\right]
$$

In a double logarithmical scale this temperature decreases first linearly with a slope of $-1 / 2$ and then tends to the constant value $T_{\text {end }}$ by the time:

$$
t_{w}=\frac{d^{2}}{\kappa \pi}
$$

It is to note that the typical time constant of the transmission measurement $\left(t_{1 / 2}\right)$ and the reflection measurement $\left(t_{w}\right)$ differ only by a constant multiplication factor, independently on the material parameters and on the thickness of the sample:

$$
t_{w}=\frac{\pi}{1.38} t_{1 / 2} \approx 2.27 t_{1 / 2}
$$

In order to calculate a 3D map, i.e. the thickness $d$ in each point of the sample, in first step the time $t_{w}$ has to be identified. It can be determined by the derivation of the temperature versus time function in double logarithmical scale $[3,16]$. The first derivative has an inflection point at time $t_{w}$ which results in a maximum of the second derivative. Fig. 5 demonstrates this behavior for a temperature function calculated using Eq.5. This equation is valid for sample with infinite extension in $x-y$ direction, which allows a onedimensional solution of the heat conductivity equation. In the case of a defect with finite size in the $x-y$ direction a 3D model calculation is required.
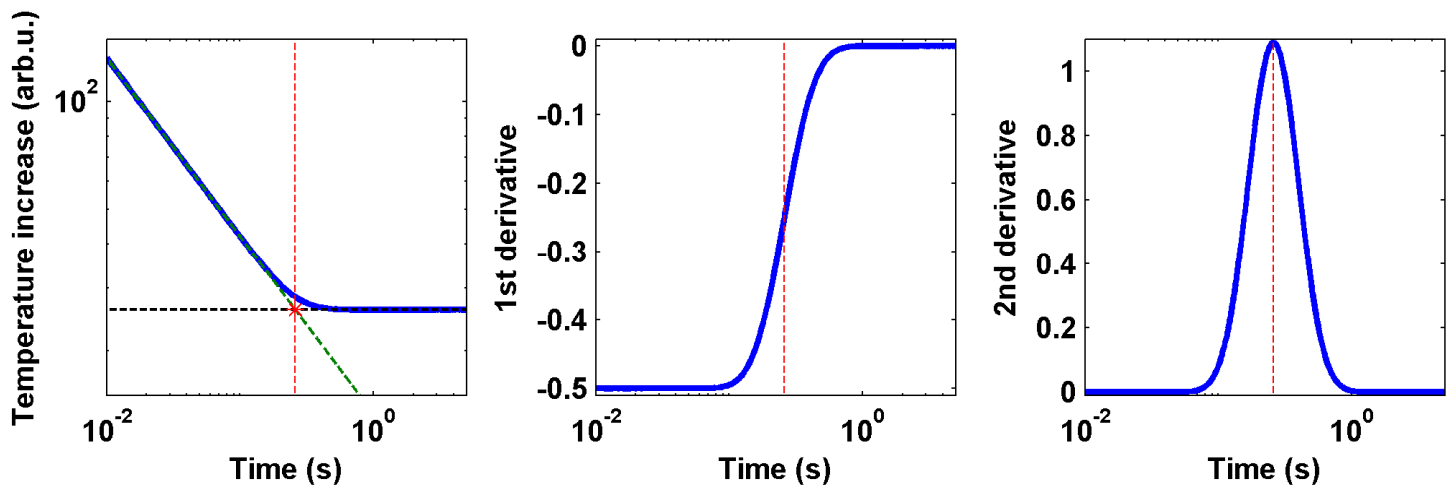

Figure 5: Temperature versus time function after a Dirac delta heating of a sample with infinite extension in $x$-y-direction and with $1 \mathrm{~mm}$ thickness in $z$-direction (left), and its $1^{\text {st }}$ (mid) and $2^{\text {nd }}$ derivative (right figure).

\section{Simulation results}

Finite element simulation models with the multi-physics simulation program of ANSYS [17] have been setup and the temperature increase above disc-shaped defects with different sizes and in different depths was investigated. In Fig. 6 simulated temperature functions and their derivatives above a defect with $10 \mathrm{~mm}$ diameter in different depths are compared with analytically calculated functions of Eq. 5 . The heat starts to accumulate above the defect at the time $t_{w}$, and this time depends only on the depth of the defect, regardless on its size, see Eq. 6 . With increasing time, due to the finite extension of the defect and due to the lateral heat diffusion the temperature decreases again $[18,19]$. This temperature 'plateau' of the heat accumulation shrinks with increasing defect depth and with smaller defect sizes, making it more and more difficult to detect these defects. E.g. for the defects in $1.5 \mathrm{~mm}$ and in $2 \mathrm{~mm}$ depth (green and black curves in Fig.6) this plateau of the temperature function is not recognisable any more. Nevertheless, the 
$2^{\text {nd }}$ derivative still shows its position, allowing of its detection. As for small and deep defects the heat accumulation and the lateral heat diffusion take place simultaneously, not only the 'plateau' of the accumulation diminishes, but also the maximum of the $2^{\text {nd }}$ derivative is shifting to shorter time compared to the theoretical $t_{w}$ value. This is e.g. the case for the maximum of the $2^{\text {nd }}$ derivative of the simulation curves (green and the black solid lines) compared to the maximum of the corresponding theoretical functions, according to Eq.5 (green and black dashed lines).

Additionally to note, that the time when the temperature decreases again due to the lateral heat diffusion depends not only on the size of the defect, but also on the lateral heat conductivity. CFRP is a very anisotropic structure, the heat conductivity of the carbon fibres in the plane is much higher than across the structure. Therefore the accumulation plateau is much narrower as it would be the case for an isotropic material. This fact makes it more difficult to localize small defects in a CFRP sample.
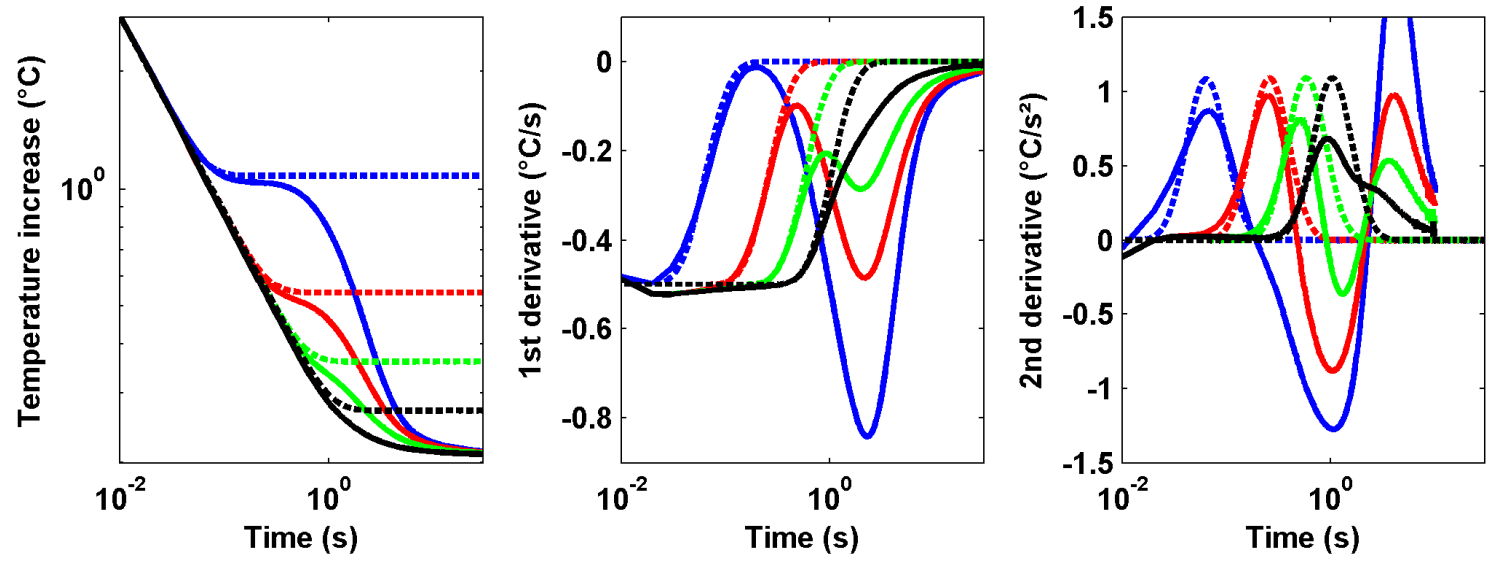

Figure 6: Temperature vs. time function and its derivatives for simulation results (solid lines) above a defect with $10 \mathrm{~mm}$ diameter and theoretical functions for infinite extension (dashed lines). Thickness of the whole CFRP model is $2.5 \mathrm{~mm}$ and the defects in a depth of $0.5 \mathrm{~mm}$ (blue line), $1 \mathrm{~mm}$ (red line, $1.5 \mathrm{~mm}$ (green line) and $2 \mathrm{~mm}$ (black line).

\section{Reflection measurements}

The CFRP structure, as described in Section 1, has been tested in reflection mode, flash heating was performed using $1 \mathrm{~ms}$ heating pulse and the temperature of the heated side was imaged with an InSb camera. Fig.7 shows the temperature change in double logarithmical scale in points lying in regions with different number of layers. In order to determine from the $2^{\text {nd }}$ derivative of measured temperature functions the time $t_{w}$, at which the accumulation starts, first a polynomial was fitted, as it is suggested by the TSR technique [3]. The big advantage of the polynomial fitting is that it reduces the noise, allowing the calculation of the derivatives. Additionally, it results in a strong data compression, as the noise reduced thermograms can be restored from the coefficients of the polynomials $[3,16]$.

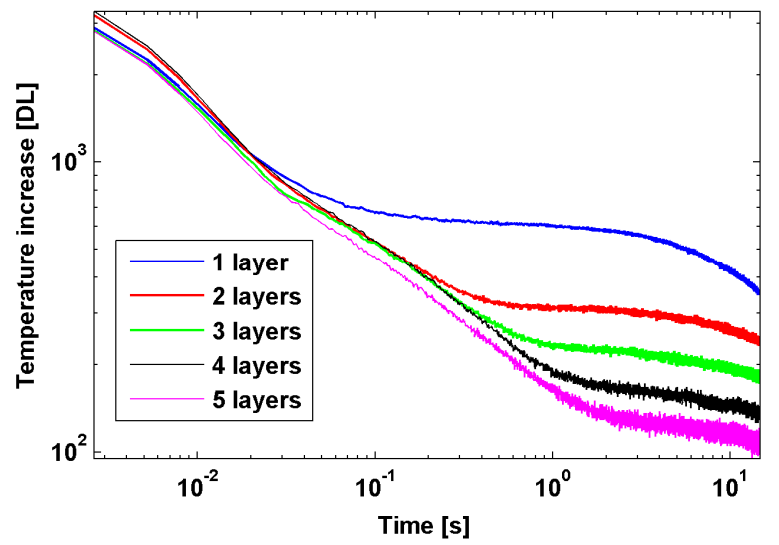

Figure 7: Temperature increase in reflection measurement for 5 different points of the CFRP sample. 
Fig. 8 shows a $2^{\text {nd }}$ derivative image calculated after the polynomial fitting of the TSR technique. In this image all the Teflon inserts, even the deepest one between the fourth and fifth layer (the upper right one) can be well recognized.

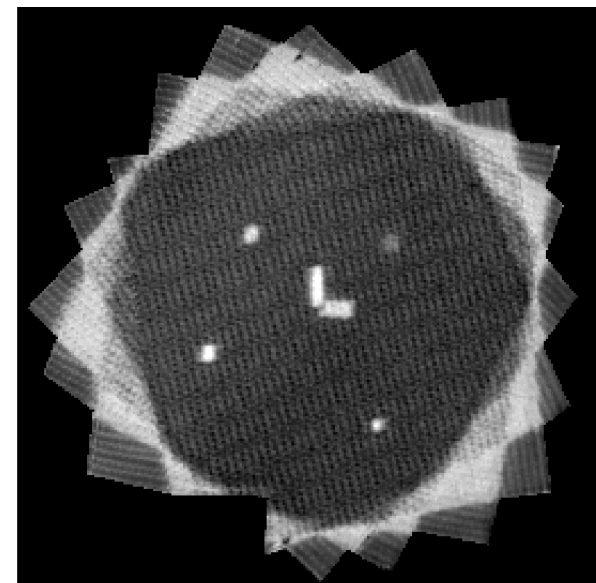

Figure 8: $2^{\text {nd }}$ derivative image calculated after TSR fitting ( $\left.t=14.6 \mathrm{~s}\right)$

The next step is to determine $t_{w}$ for each pixel from the derivatives and then calculate a 3D map. By closer investigation of the temperature function in couple of cases a significant difference can be observed between the $t_{w}$ values obtained from the maximum of the $2^{\text {nd }}$ derivative of the theoretical function and of the transmission measurements. Such a case is depicted in Fig. 9 for a point lying in a region with only 1 layer thickness. The measurement results differ from the theoretical values because of two main reasons:

- $\quad$ The heating pulse is not instantaneous, but a short (about $1 \mathrm{~ms}$ long) pulse. Therefore the temperature in double logarithmical scale shortly after the pulse does not decrease with a slope of $-1 / 2$, but less.

- $\quad$ The conditions are not adiabatic; because of heat loss to the environment the temperature does not remain constant but decreases further.
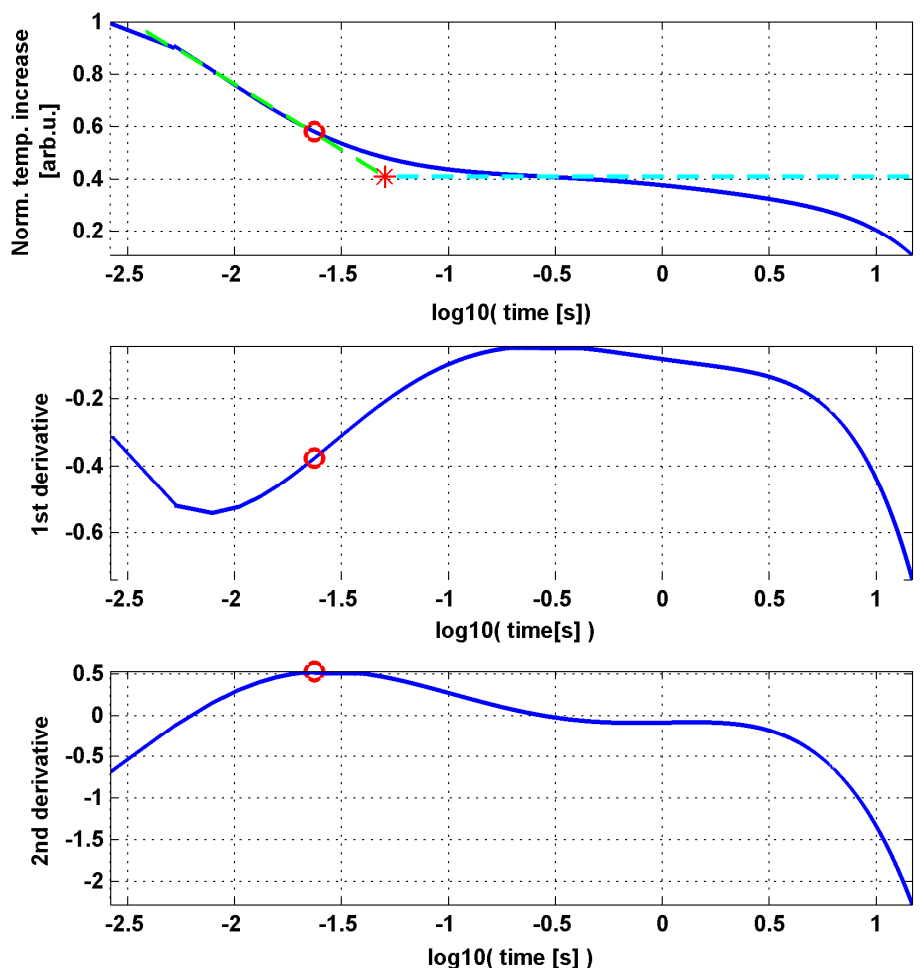

Figure 9: Measured temperature function in a point lying in a region with only one CFRP layer (thickness $0.5 \mathrm{~mm}$ ) after polynomial fitting (top figure) and its $1^{\text {st }}$ (mid) and $2^{\text {nd }}$ (bottom figure) derivatives. The red circle marks the maximum of the $2^{\text {nd }}$ derivative. To the first part of the temperature function a line with a slope of $-1 / 2$ is fitted (green dashed line) and to the temperature plateau ( $1^{\text {st }}$ derivative close to 0 ) a line with constant temperature value (violet dashed line). The intersection of both lines (marked by red star) is taken as the corrected $t_{w}$ value. 
In order to minimize the influence of these two factors an additional step is applied to determine the $t_{w}$ value: a straight line with a slope equal to $-1 / 2$ is fitted to the $1^{\text {st }}$ part of the curve (green dashed line in Fig. 9), and a horizontal line is added to the $2^{\text {nd }}$ part of the function (violet dashed line in Fig. 9), where the temperature function has an inflection point, that means where the $1^{\text {st }}$ derivative is close to zero. For a corrected $t_{w}$ the intersection point of these two lines is taken (marked by a red star) instead of the maximum of the $2^{\text {nd }}$ derivative (marked by a red circle). This corrected value is much closer to the theoretically expected value, while the two main errors of the measurement (listed above) are corrected. This modification has been found to be necessary only for very thin parts of the sample, that means only for areas, where the sample consists of only one layer. In the other parts with 2-5 layers the results of the modified calculation are very similar to the original one, obtained by the maximum of the $2^{\text {nd }}$ derivative.

Fig. 10 shows the 3D map of the sample calculated by this technique. It can be seen that the thicknesses of all the areas, where 1,2,3,4 or 5 carbon fibre layers lie on each other, could be determined excellently. The depths of the Teflon inserts are calculated partially correctly: the depth of the highest inserts (square in the lower right corner and the vertical strip in the middle, see also in the middle of Fig. 2) and the second shallowest defect (lower left corner) is determined properly. The depth of the inserts between the $3^{\text {rd }}$ and $4^{\text {th }}$ layers, the square in the upper left corner and the horizontal stripe in the middle (see also at the photo in the right of Fig.2) are well visible in the depth map, but not all the pixels got a correct depth value. The depth of the deepest artificial defect (upper right corner) is hardly recognisable in the 3D depth map.
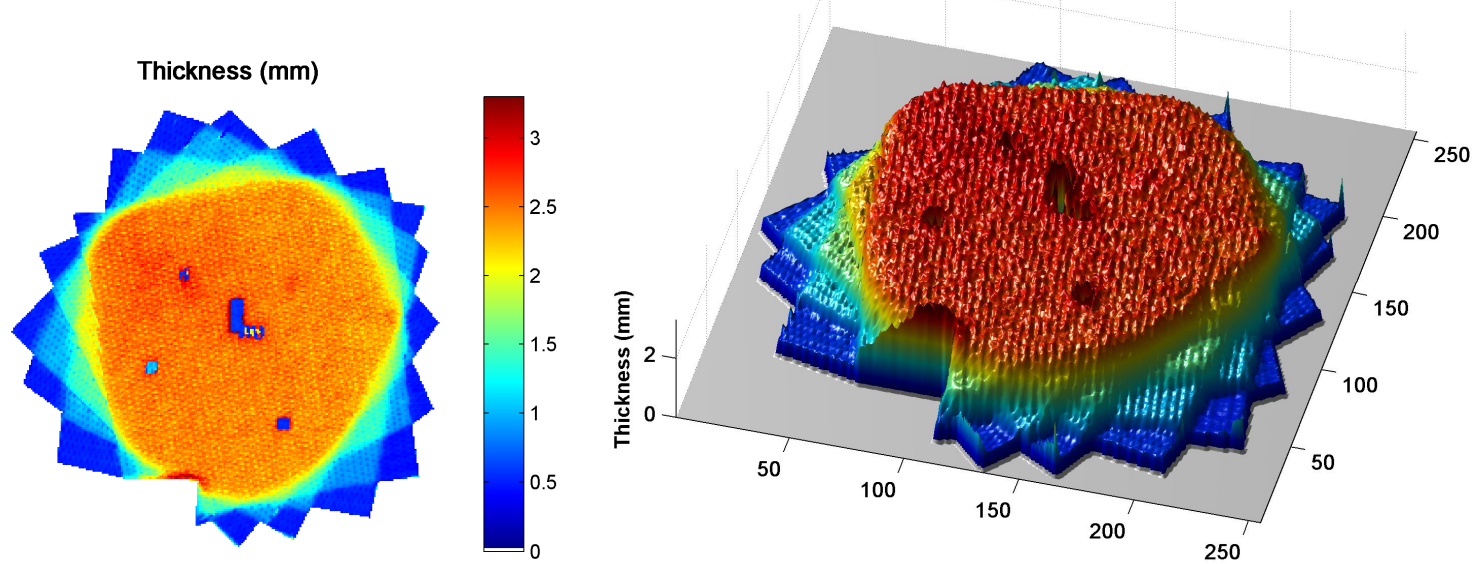

Figure 10: Calculated 3D depth map of the sample.

\section{Summary}

It has been shown that using the TSR technique, after polynomial fitting of the temperature function in double logarithmical scale, the maximum of the 2nd derivative can be used to determine the depth of defects and the thickness of the sample. For thin and shallow parts, where the non-instantaneous heating of the sample and the non-adiabatic circumstance significantly affect the measurement results, additional correction by fitting two lines is suggested. While the accumulation plateau of the temperature curve is fully diminished, the depth of small and deep defect could not be determined properly, even if the defect itself is well recognisable in the $2^{\text {nd }}$ derivative image.

\section{Acknowledgment}

I would like to thank Steve Shepard for the fruitful discussions.

\section{REFERENCES}

[1] Busse G., Wu D., Karpen W., Thermal wave imaging with phase sensitive modulated thermography, J. Appl. Phys. 71(8), 1992.

[2] Maldague X., Marinetti S., Pulse Phase Infrared Thermography, J. Appl.Phys., Vol. 79, No. 5, pp. 2694-2698, 1996.

[3] Shepard, S.M., "Temporal noise reduction, compression and analysis of thermographic image data sequences," U.S. Patent 6516084, (2003)

[4] Ibarra-Castanedo, C., Avdelidis, N.P., Grinzato, E.G., Bison, P.G., Marinetti, S., Chen, L., Genest, M., Maldague, X.,"Quantitative inspection of non-planar composite specimens by pulsed phase thermography", QIRT Journal 3(1), 25-40 (2006). 
[5] Wallbrink C., Wade S.A., Jones R., "The effect of size on quantitative estimation of defect depth in steel structures using lock-in thermography", J. Appl. Phys. 101(10), 104907-8 (2007).

[6] Pickering S. and Almond D., "Matched excitation energy comparison of the pulse and lock-in thermography NDE techniques", NDT\&E International 41, 501-509 (2008).

[7] Balageas D.L. "Defense and illustration of time-resolved pulsed thermography for NDE", QIRT Journal, 9:1, 3-32, (2012) DOI:10.1080/17686733.2012.676902

[8] Sankar, B.V. Low-velocity impact response and damage in composite materials. Key Eng. Mater. 1996, 120-121, 549-582.

[9] Bhattacharyya, D.; Fakirov, S. Synthetic Polymer-Polymer Composites; Carl Hanser Verlag: München, Germany, 2012.

[10] Mitschang, P.; Neitzel, M. Handbuch Verbundwerkstoffe; Carl Hanser Verlag: München, Germany, 2004.

[11] Breuer, U.; Neitzel, M. Die Verarbeitungstechnik der Faser-Kunststoff-Verbunde; Carl Hanser Verlag: München, Germany, 1997.

[12] Maier A., Schmidt R., Oswald-Tranta B., Schledjewski R., " Non-Destructive Thermography Analysis of Impact Damage on Large-Scale CFRP Automotive Parts"; Materials 2014, 7(1), 413429; 2014.

[13] Carslaw H.S., Jaeger J.C., Conduction of Heat in Solids, Oxford University Press, Oxford, 1959.

[14] Parker W.J., Jenkins R.J., Butler C.P., Abbott G.L., "Flash method of determining thermal diffusivity, heat capacity and thermal conductivity", J. Applied Physics, Vol. 32, pp. 1679-1684, Sept. 1961.

[15] FLIR Systems, Inc., http://www.flir.com

[16] Shepard, S. M., "Advances in thermographic NDT", SPIE Proc. 5074, 882-887 (2003).

[17] ANSYS Inc., http://www.ansys.com

[18] Shepard S. M., Lhota J. R., Ahmed T., Flash thermography contrast model based on IR camera noise characteristics, J. Nondestructive Testing and Evaluation, 2007.

[19] Oswald-Tranta B., Shepard S.M. „Comparison of Pulse Phase and Thermographic Signal Reconstruction Processing Methods“, in Proc. SPIE, Thermosense XXXV, vol.8705 (2013). 